East Tennessee State University

Digital Commons@ East Tennessee State University

ETSU Faculty Works

Faculty Works

6-1-1997

\title{
Auditory Filters Measured at Neighboring Center Frequencies
}

Marc A. Fagelson

East Tennessee State University, fagelson@etsu.edu

C.A. Champlin

University of Texas

Follow this and additional works at: https://dc.etsu.edu/etsu-works

Part of the Speech Pathology and Audiology Commons

\section{Citation Information}

Fagelson, Marc A.; and Champlin, C. A.. 1997. Auditory Filters Measured at Neighboring Center Frequencies. The Journal of the Acoustical Society of America. Vol.101(6). 3658-3665. https://doi.org/10.1121/1.418326 ISSN: 0001-4966

This Article is brought to you for free and open access by the Faculty Works at Digital Commons @ East Tennessee State University. It has been accepted for inclusion in ETSU Faculty Works by an authorized administrator of Digital Commons @ East Tennessee State University. For more information, please contact digilib@etsu.edu. 


\section{Auditory Filters Measured at Neighboring Center Frequencies}

\section{Copyright Statement}

Copyright 1997 Acoustical Society of America. This article may be downloaded for personal use only. Any other use requires prior permission of the author and the Acoustical Society of America.

The following article appeared in Marc A. Fagelson, Craig A. Fagelson, Measurement of Auditory-Filters at Neighboring Center Frequencies, Journal of Acoustical Society of America,101, 3658 (1997) and may be found at https://doi.org/10.1121/1.418326. 


\title{
Auditory filters measured at neighboring center frequencies ${ }^{\text {a) }}$
}

\author{
Marc A. Fagelson \\ Department of Communicative Disorders, East Tennessee State University, Johnson City, Tennessee 37614
}

Craig A. Champlin

Department of Communication Sciences and Disorders and Institute for Neuroscience, University of Texas, Austin, Texas 78712

(Received 12 April 1996; accepted for publication 14 January 1997)

\begin{abstract}
Auditory filters were derived in 20 normal-hearing human listeners at center frequencies (CFs) of 913, 1095, 3651, and $4382 \mathrm{~Hz}$ using the roex $(p, r)$ method. Comparisons were made between slopes of the filters' skirts at the neighboring CFs with filter output levels of 45 and $70 \mathrm{~dB}$. The same comparisons were made with regard to filter equivalent rectangular bandwidth (ERB). In the $1000-\mathrm{Hz}$ region, the low-frequency slopes $(\mathrm{Pl})$ of filters centered at 913 and $1095 \mathrm{~Hz}$ were significantly correlated at both stimulus levels, while the high-frequency slopes $(P u)$ were similar only at the high test level. In the 4000-Hz region, for sinusoids of 3651 and $4382 \mathrm{~Hz}$, the level effect was clearer as both $P u$ and $P l$ values diverged at the low level but were related at high levels. The ERBs centered at the same CFs displayed a similar level dependence. At the stimulus level most likely to be affected by an active feedback mechanism, auditory filters centered at nearly the same frequency displayed quite distinct frequency selectivity, and this trend was stronger in the $4000-\mathrm{Hz}$ region than the $1000-\mathrm{Hz}$ region. The findings suggest that a saturating, active cochlear mechanism may not be distributed evenly, or contribute to peripheral tuning with equal effectiveness throughout the length of the partition. (c) 1997 Acoustical Society of America. [S0001-4966(97)03805-8]
\end{abstract}

PACS numbers: 43.66.Dc [WJ]

\section{INTRODUCTION}

A primary function of the mammalian ear is to analyze the spectral components of complex sounds produced by vibrations propagated through an appropriate medium. As Gold and Pumphrey (1948) pointed out, a nervous system comprised of cells that could instantaneously encode acoustic occurrences would obviate the need for a sensory organ that analyzed frequency. Because the nervous system is able to transmit a finite amount of information per unit time, and the pressure fluctuations of an acoustic signal are often too rapid to be faithfully encoded by the nervous system, there is a distinct need for the sensory organ, in this case the cochlea, to analyze a significant portion of the acoustic energy's spectrum.

Although Gold (1948) indicated that the sharp tuning of the healthy cochlea required an active component, several points remain unclear regarding the nature of the "activeness." First, it would be beneficial to know approximately the amount of energy provided by the feedback mechanisms relative to some input level. Second, it is possible that, because structures along the cochlear partition vary morphologically in basal and apical regions, the relative contributions of an active feedback system would differ when tuning is measured across areas of the cochlea. Third, as the active processing requires an external energy source, the structures that utilize the energy may lose their active properties when damaged or otherwise metabolically compromised.

Addressing the first point is beyond the scope of this research and is currently a source of debate among research-

\footnotetext{
${ }^{a)}$ Portions of this paper were presented at the 130th meeting of the Acoustical Society of America [J. Acoust. Soc. Am. 98, 2945 (A) (1995)].
}

ers (e.g., Allen and Fahey, 1992). The second issue, as with the first, has a direct impact on the frequency-analyzing power exhibited throughout the cochlea and may be investigated psychophysically by testing a listener's performance on a frequency-resolution task that reflects, to some degree, the integrity of the cochlea's active process. The third point suggests that the active mechanical response is physiologically vulnerable and that its loss at discrete places along the cochlear partition could produce auditory filters that differ markedly. Moreover, Mills and Rubel (1996) demonstrated the contribution such mechanisms contribute to the production of distortion-product otoacoustic emissions (DPOAEs). The research reported here focused on the latter two points.

The active feedback thought to exist along the cochlear partition is evinced by a nonlinear component superimposed upon the otherwise passive motion of the basilar membrane, and level dependencies clearly exist in the behavior of this nonlinear component. Zwicker (1979) characterized the active process as one that saturated at input levels around 40 $\mathrm{dB}$, and physiological measurements of the cochlear partition's motion have shown that the nonlinearity's relative contribution to the overall basilar-membrane motion is smallest at high stimulus levels. Psychophysically, such changes may be observed as the widening of auditory filters and concurrent decreases in the slopes of the auditory filters' low-frequency skirts (e.g., Formby, 1990; Moore and Glasberg, 1983; Patterson, 1976; Rosen and Baker, 1994; Rosen and Stock, 1992; Sommers and Humes, 1993; Weber, 1977). However, the effect of stimulus level at different center frequencies (CFs) is less clear. Although Weber (1977) demonstrated that the auditory filter is affected by stimulus level to essentially the same degree across several CFs each separated by an octave, Rosen and Stock reported that, as CF was 
raised from 125 to $1000 \mathrm{~Hz}$, an increase in masker level produced a relatively greater change in filter parameters. Rosen and Stock attributed their findings to the possibility that the cochlea's nonlinear behavior was less pronounced at the apex. It should be noted that filters centered at adjacent (i.e., <1/3 octave) CFs have not been compared as extensively as those centered an octave apart.

A study comparing filter selectivity at neighboring CFs could address the level dependence of the active feedback process and its relative contribution at different places along the cochlear partition. Two auditory filters may exhibit similar skirt slopes at one test level and therefore filter sound with similar effectiveness. At a different intensity the slopes may diverge from one another on the low- and/or highfrequency side. The level manipulation would change cochlear tuning by altering the relative contribution of a saturating active process whose presence affects basilar membrane damping primarily in a narrow region around the location of a traveling wave's maximum displacement (e.g., Dallos, 1992). If cochlear amplification is distributed unevenly throughout the length of the partition, a manipulation of test level may exert similar, but not identical, effects on tuning at different $\mathrm{CFs}$.

It is assumed that passive basilar membrane mechanics are more resistant to physical trauma than the active feedback process theorized by Gold (1948). Evidence for the vulnerability of active mechanics has been demonstrated using physiological techniques (e.g., Rhode, 1971, 1977; Robles et al., 1986), and the measurement of DPOAEs (e.g., Whitehead et al., 1992). Additionally, Subramaniam et al. (1994) indicated that the physiological vulnerability of DPOAEs varied across frequency region when measured before, during, and after a regimen of noise exposure. Specifically, after it was abolished by intense broadband noise, DPOAE amplitude returned to pre-exposure levels in the $1000-\mathrm{Hz}$ region, but not the $4000-\mathrm{Hz}$ region, despite total outer hair cell loss at both places. Emissions were particularly susceptible to the effects of noise when evoked by lowlevel primaries at the $4000-\mathrm{Hz}$ place. One conclusion drawn from this work was that the outer hair cells contributed relatively more to the production of emissions in the $4000-\mathrm{Hz}$ region than in the $1000-\mathrm{Hz}$ region.

Psychophysical experiments have focused on the derivation of auditory-filter parameters at one or more frequencies, but the CFs examined typically differed by at least an octave. It is suggested that filter parameters derived using low-level maskers should be more dependent upon the active mechanics than those obtained with high-level maskers. Differences in processing across slightly different CFs, then, may provide additional evidence of an active feedback system if proximate-CF filters differ only at the low levels.

\section{METHOD}

\section{A. Subjects}

Auditory-filter shapes were determined for 20 adult humans [ 13 females and 7 males; ages 21 to 40 years old (mean $=26.8 \mathrm{yr}$ )] who reported no significant exposure to occupational or recreational noise, and no regular medication with ototoxic drugs. All listeners exhibited absolute sensitivity within $15 \mathrm{~dB}$ of the ANSI (1989) standard for air-conduction thresholds when screened at octave frequencies from 250 through $8000 \mathrm{~Hz}$.

An additional test using a Bekesy sweep-frequency technique was conducted to assess sensitivity at interoctave frequencies, such as those at which the auditory filters were centered. The Bekesy recording was completed by 15 of the test subjects and confirmed normal absolute sensitivity. The remaining five subjects were not available for testing when the Bekesy procedure was in operation. The Bekesy tracking was conducted at a one octave/minute rate and so a determination of threshold microstructure was not made (e.g., Long, 1984).

Subjects' middle ear status was screened prior to the first test session. The screening procedure included only a measure of tympanic membrane compliance and all test subjects demonstrated normal middle ear function (e.g., Margolis and Shanks, 1985). Acoustic reflexes were not evaluated, as the central nervous system component attributed to normal reflex function was not of interest in this research.

Test ear was determined by screening subjects for spontaneous otoacoustic emissions (SOAEs) in an effort to preclude the possibility that such an emission could interact with a very low-level signal at a test frequency (e.g., Pasanen et al., 1987). Two potential subjects were excluded due to the presence of SOAEs bilaterally within $250 \mathrm{~Hz}$ of at least one of the CFs. Four subjects who participated in the study displayed SOAEs at or near a CF in the left ear. These individuals were then screened in the right ear, which, when it did not display SOAEs, was used as the test ear. The left ear was used in all remaining subjects.

\section{B. Instrumentation}

Masked thresholds were determined with the subjects seated in a sound-treated booth and listening through TDH-49 headphones mounted in MX/41-AR cushions. Tonal signals and maskers were generated digitally at a sampling rate of $25 \mathrm{kHz}$ using a Tucker-Davis Technologies QDA1 board with QAP1 array processor. Test signals were lowpass filtered at $5 \mathrm{kHz}$, while maskers were low-pass filtered at $10 \mathrm{kHz}$ using a TTE1 1629 FSB filter system. Roughly one-half of the subjects were run with the masking noise filtered using a Rockland 751A Brickwall. The latter filter malfunctioned during data acquisition, prohibiting its use with all subjects and requiring two listeners, JL and TUM, to repeat 3 and 12 runs, respectively. The TTE filter was used for both signal and masker filtering following the Rockland malfunction.

The generation and presentation of the stimuli and the tracking of subjects' responses were accomplished by a personal computer (PC). Its monitor and keyboard were located inside the test booth.

\section{Stimuli}

Tonal signals at frequencies of 913, 1095, 3651, and $4382 \mathrm{~Hz}$ were presented in a simultaneous-masking context. 
TABLE I. The four different masker cutoffs (LP=low-pass cutoff; HPhigh-pass cutoff, both in $\mathrm{Hz})$. A broadband noise masker $(0-10 \mathrm{kHz})$ was also used to establish the filters' level of operation.

\begin{tabular}{ccccc}
\hline \hline Filter CF & $\begin{array}{c}\mathrm{CF}( \pm 0.2) \\
\text { LP/HP }\end{array}$ & $\begin{array}{c}\mathrm{CF}( \pm 0.4) \\
\text { LP/HP }\end{array}$ & $\begin{array}{c}\mathrm{CF}(-0.2,0.4) \\
\text { LP/HP }\end{array}$ & $\begin{array}{c}\mathrm{CF}(-0.4,0.2) \\
\text { LP/HP }\end{array}$ \\
\hline $913 \mathrm{~Hz}$ & $730 / 1096$ & $548 / 1461$ & $730 / 1461$ & $548 / 1096$ \\
$1095 \mathrm{~Hz}$ & $876 / 1314$ & $657 / 1533$ & $876 / 1533$ & $657 / 1314$ \\
$3651 \mathrm{~Hz}$ & $2920 / 4391$ & $2189 / 5121$ & $2920 / 5121$ & $2189 / 4391$ \\
$4382 \mathrm{~Hz}$ & $3486 / 5238$ & $2609 / 6115$ & $3486 / 6115$ & $2609 / 5238$ \\
\hline \hline
\end{tabular}

These frequencies were selected because, in a related study (Fagelson and Champlin, 1996), they served as primary tones during the measurement of DPOAEs. Signals and maskers were synchronously gated on and off with total durations of $400 \mathrm{~ms}$, including 10-ms rise-fall times.

Masker conditions included a broadband $(0-10 \mathrm{kHz})$ noise, which was used in both the growth-of-masking and the auditory-filter shape tests, and four additional conditions in which symmetric and asymmetric notches were imposed on the noise. Notch-noise conditions included the cut-off frequencies indicated in Table I.

Masking noise was used to determine the auditory-filter shape at each test frequency as masked thresholds were fitted to the roex $(p, r)$ model developed by Patterson (1976). Stone et al. (1992) have demonstrated a five-point roex method in which fewer maskers than were used by Patterson produced essentially the same measures of filter bandwidth and symmetry. Therefore, in the current research, a degree of resolution in the delineation of auditory-filter shapes was sacrificed for the time required to test a large pool of listeners.

\section{Procedure}

Signal level was adapted using a two-interval, forcedchoice procedure with feedback to target $70.7 \%$ correct detection of the signal (Levitt, 1971). Subjects were presented with a visual prompt ("ready"), two intervals marked by signal lights, and a "respond" prompt. Subjects were allowed as much time as they needed to respond. Subjects responded with a keystroke appropriate to the interval in which they heard the test signal. In all testing, the mean of at least three runs was used to estimate threshold. Each run consisted of 50 trials and was retained only when the standard deviation (s.d.) within that run was less than $4.0 \mathrm{~dB}$. The standard errors across the mean of runs for each masker condition, from which auditory-filter shape parameters were calculated, were less than $3.0 \mathrm{~dB}$. For those conditions producing standard errors greater than $3.0 \mathrm{~dB}$, at least two additional runs were completed in order to decrease withincondition variance to the $3.0-\mathrm{dB}$ criterion level. Additional runs were necessary in at least one and not more than five out of the ten conditions completed by all subjects. The broadband condition was tested first, the symmetric-notch conditions second, and the asymmetric-notch conditions last. More than $75 \%$ of all conditions resulted in standard errors of $<2.0 \mathrm{~dB}$.

Prior to the acquisition of auditory-filter shape information, a growth-of-masking function was obtained using sig-
TABLE II. Mean $(N=20)$ relative levels of operation for the auditory filters centered at the four primary-tone frequencies, and two primary-tone levels. The target primary-tone levels were 45 and $70 \mathrm{~dB}$ SPL.

\begin{tabular}{rcccc}
\hline \hline $\begin{array}{c}\text { Auditory } \\
\text { filter CF }\end{array}$ & Low level & s.d. & High level & s.d. \\
\hline $913 \mathrm{~Hz}$ & 44.94 & 2.31 & 70.23 & 2.74 \\
$1095 \mathrm{~Hz}$ & 45.56 & 2.15 & 70.64 & 3.19 \\
$3651 \mathrm{~Hz}$ & 45.33 & 2.56 & 71.74 & 3.61 \\
$4382 \mathrm{~Hz}$ & 46.97 & 1.98 & 72.99 & 3.21 \\
\hline \hline
\end{tabular}

nal frequencies of 1000 or $4000 \mathrm{~Hz}$ presented simultaneously with broadband noise. Pressure spectrum levels for these maskers were $0,10,20$, and $30 \mathrm{~dB}$ SPL/Hz. A regression line was fitted to the four masked thresholds obtained from each listener at each signal frequency. Using the line's slope and $y$ intercept, the masker levels were adjusted for each subject to produce broadband masked thresholds of either $45 \mathrm{~dB}$ SPL or $70 \mathrm{~dB}$ SPL. These thresholds were equated across subjects and were considered outputs of auditory filters (e.g., Rosen and Baker, 1994) in the wideband masker, or reference condition. The ranges of spectrum levels were 24-32 and $45-56 \mathrm{~dB}$ in the $1000-\mathrm{Hz}$ region and $18-26$ and $41-49$ $\mathrm{dB}$ in the $4000-\mathrm{Hz}$ region for the low and high levels, respectively. Obtaining the growth-of-masking functions familiarized the subjects with the test paradigm, providing at least four hours of practice time for each subject. Additionally, about half the subjects had never participated in a psychophysical experiment, and these persons practiced for two hours prior to the growth-of-masking test. Mean broadbandmasked thresholds are listed in Table II and were, in 66 out of the 80 frequency-by-level conditions $(82.5 \%)$, within $3 \mathrm{~dB}$ of the desired filter output levels.

\section{E. Data analysis}

The slopes of auditory-filter skirts [designated in the roex $(p, r)$ procedure as $P l$ and $P u$ for the low- and highfrequency skirts, respectively] were determined using a program written for the PC by Glasberg and Moore (1990). The $P l$ and $P u$ of the filters in the $1000-\mathrm{Hz}$ region are depicted schematically in Fig. 1. In the slope correlations, a slope of one of the filter's skirts, say $P l$, is measured at two neighboring CFs (e.g., 913 and $1095 \mathrm{~Hz}$ ) and the two values are correlated across the 20 subjects. The same comparison is made for the $P u$ values, as well as both sets of skirt slopes in the $4000-\mathrm{Hz}$ region. Equivalent rectangular bandwidth (ERB) of the auditory filter is calculated from the slope information, and comparisons are made between ERBs of filters centered at neighboring CFs at the two stimulus levels.

An additional filter parameter, symmetry of the auditory filter, was determined by dividing the high-frequency slope by the low-frequency slope, producing the so-called symmetry index $(\mathrm{SI}=P u / P l)$ (e.g., Patterson et al., 1982; Sommers and Humes, 1993). An SI of 1.0 corresponds to a filter whose low- and high-frequency slopes are identical, consistent with a symmetric filter shape. A symmetry measure of less than 1.0 indicates the low-frequency slope of the filter is steeper than the high-frequency slope, while a quotient greater than 


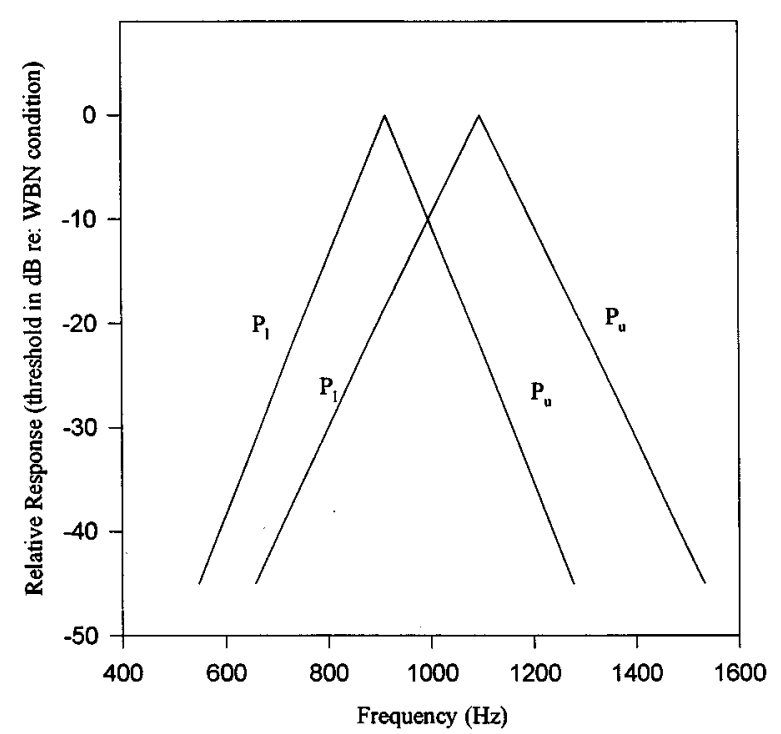

FIG. 1. Schematic representation of neighboring auditory filters centered in the $1000-\mathrm{Hz}$ region. The correlations between the two $\mathrm{Pl}$ values are calculated in the $1000-\mathrm{Hz}$ region and the $4000-\mathrm{Hz}$ region, at both masker levels. The correlations between the $P u$ values are also determined. Additionally, the symmetry index $(\mathrm{SI})$ is determined by obtaining the quotient $P u / P l$ for each of the filters.

1.0 indicates a filter whose high-frequency slope is steeper than its low-frequency slope.

\section{RESULTS}

\section{A. Low- and high-frequency slopes of the auditory filter}

Mean slopes and standard deviations of the auditory filters centered at the four CFs appear in Table III. Additional reanalyses (see below) of slope data appear in the Appendix along with the mean values from Table III for comparison. Larger values indicate steeper slopes. Note that $P l$ decreased substantially, while $P u$ increased slightly with level. A fourway analysis of variance (ANOVA) (frequency region, CF, level, slope) using the Geisser-Greenhouse correction (GGF) for the repeated measures obtained from each subject, revealed that no main effects were significant. That is, regardless of frequency region tested, the filter slopes behaved in a similar manner when assessed at the different levels and CFs. Therefore, the slope values could be collapsed across frequency to provide the overall mean data presented in Fig. 2. The figure shows that at the low level, the low-frequency

TABLE III. Mean $(N=20)$ low- and high-frequency auditory-filter slopes with one standard deviation ( $P l$ and $P u$, respectively).

\begin{tabular}{|c|c|c|c|c|c|c|c|c|}
\hline & \multicolumn{2}{|c|}{$913 \mathrm{~Hz}$} & \multicolumn{2}{|c|}{$1095 \mathrm{~Hz}$} & \multicolumn{2}{|c|}{$3651 \mathrm{~Hz}$} & \multicolumn{2}{|c|}{$4382 \mathrm{~Hz}$} \\
\hline & $P l$ & $P u$ & $P l$ & $P u$ & $P l$ & $P u$ & $P l$ & $P u$ \\
\hline \multicolumn{9}{|c|}{ Low level } \\
\hline Mean & 29.2 & 22.8 & 26.5 & 26.2 & 28.3 & 24.8 & 32.4 & 25.7 \\
\hline s.d. & 4.66 & 4.83 & 4.53 & 5.59 & 4.25 & 5.91 & 7.50 & 5.78 \\
\hline \multicolumn{9}{|c|}{ High level } \\
\hline Mean & 24.9 & 26.7 & 21.3 & 29.1 & 21.6 & 28.1 & 21.4 & 30.5 \\
\hline s.d. & 4.04 & 5.82 & 3.90 & 6.88 & 4.24 & 6.54 & 4.00 & 6.67 \\
\hline
\end{tabular}

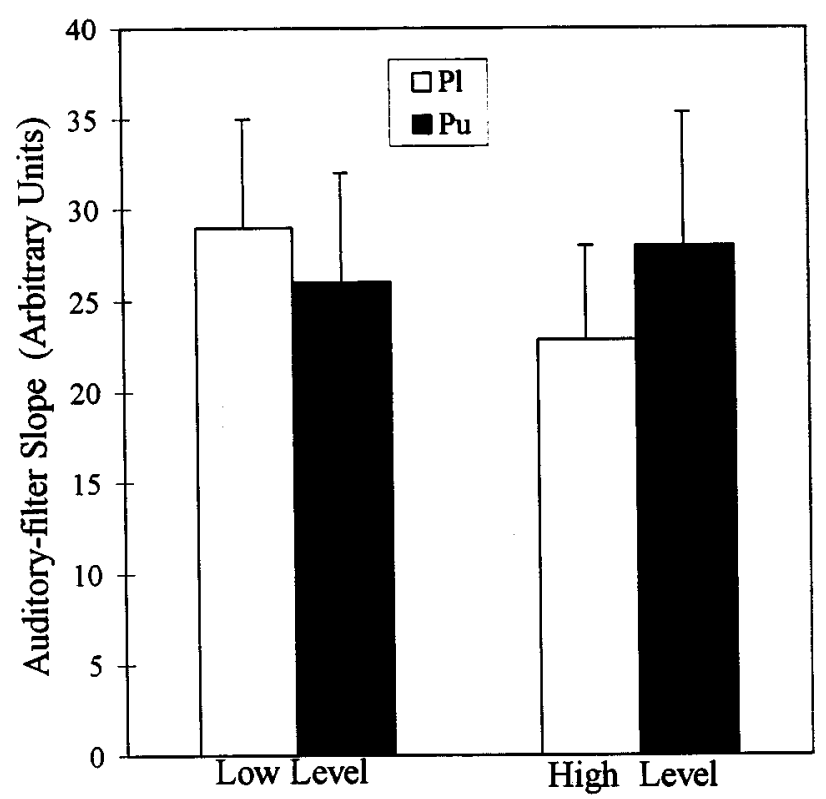

FIG. 2. Mean auditory-filter slopes assessed in both the $1000-\mathrm{Hz}$ and $4000-\mathrm{Hz}$ regions. The open bars reflect low-frequency slopes for all filters, the filled bars represent the high-frequency slopes for all filters. The $x$ axis indicates the two level conditions. Error bars indicate one standard deviation.

slope is steeper than the high-frequency slope, while the reverse is true for the two slopes at the high level. The implications for filter symmetry are identified below.

The ANOVA revealed three significant interactions, one of which included the level variable. This was the interaction between level and slope of the auditory filter illustrated in Fig. 2, which was by far the strongest effect [GGF $(1,19)$ $=2220.25 ; p<0.0001]$. The level effect is well-documented in studies of auditory-filter slope, regardless of the procedure used to derive the filter (e.g., Formby, 1990; Rosen and Baker, 1994; Weber, 1977). In the present research, the effect was associated with the slope tested, and the lowfrequency slopes were affected more than the high-frequency slopes.

The remaining two significant interactions were associated with the small change to the filter centered at $913 \mathrm{~Hz}$ when level was changed. The two interactions, $\mathrm{CF} \times$ slope $[\mathrm{GGF}(1,19)=6.262 ; p<0.05]$ and frequency region $\times \mathrm{CF}$ $\times$ slope $[\operatorname{GGF}(1,19)=10.551 ; p<0.01]$, reflected the finding that $P l$ and $P u$ values of the $913-\mathrm{Hz}$ filter changed by nearly the same amount when masker level was increased. It is not clear why the 913-Hz CF filter behaved differently from the other filter in its region $(\mathrm{CF}=1095 \mathrm{~Hz})$.

Table IV contains the correlations between slopes of the auditory-filter skirts at neighboring CFs (i.e., $P l$ at $913 \mathrm{~Hz}$ with $P l$ at $1095 \mathrm{~Hz}$ at the low stimulus level; $P l$ at $3651 \mathrm{~Hz}$ with $P l$ at $4382 \mathrm{~Hz}$ at the low stimulus level, etc.) for each frequency and level condition. The correlations were considered better measures of filter-slope similarity than a comparison of $P l$ and $P u$ mean values at the neighboring CFs because the variability created a situation in which most mean slopes were indistinguishable. Eight correlations are reported, each depicting a measure of the similarity between 
TABLE IV. Correlations tested in the two frequency regions. The association is measured between $P l$ values at each stimulus level at neighboring CFs. The same comparison is made for the $P u$ values.

\begin{tabular}{lccccc}
\hline \hline & \multicolumn{2}{c}{$1000 \mathrm{~Hz}$} & & \multicolumn{2}{c}{$4000 \mathrm{~Hz}$} \\
\cline { 2 - 3 } \cline { 5 - 6 } & $P l$ & $P u$ & & $P l$ & $P u$ \\
\hline Low level & 0.53 & 0.24 & & -0.01 & -0.18 \\
High level & $0.64^{\mathrm{a}}$ & $0.70^{\mathrm{a}}$ & & $0.83^{\mathrm{a}}$ & 0.44 \\
\hline \hline
\end{tabular}

${ }^{\mathrm{a} p} p<0.01$.

either set of slope values in the two frequency regions at the two levels. Statistical significance ( $p<0.01$ at $r= \pm 0.55$; degree of freedom $=19$ ) was found for three comparisons at the high masker level.

The conservative alpha level was chosen to reduce the possibility that a type I error would occur due to the number of correlations (eight) calculated with the same variables ( $P l$ and $P u$ ) (e.g., Glantz and Slinker, 1990). The correlations were always stronger at high signal levels than at low levels for each of the frequency regions. A significant correlation indicated that the respective slopes $(P l$ or $P u)$ for the two frequencies were closely related, or filtered the stimuli with about the same effectiveness. Thus, at the higher intensity, the auditory filters centered at neighboring frequencies assumed a more uniform shape than when assessed at the low intensity, particularly on the low-frequency side of the filter.

\section{B. Auditory-filter ERB}

The mean ERBs, expressed as a proportion of CF, appear in Table V. As for slope values, data from the reanalyses (see below) appear in the Appendix with the mean values from Table $\mathrm{V}$ for comparison. With the exception of $\mathrm{CF}$ $=913 \mathrm{~Hz}$, ERBs were larger at high test levels. A two-factor, repeated-measures ANOVA indicated the differences across level were significant at the remaining three CFs $[\operatorname{GGF}(1,19)$ $=6.19 ; p<0.05]$. No main effect for frequency was found. In general, the filter bandwidths reported here were slightly wider than those reported elsewhere.

A series of correlations similar to that described above for the slope data was then conducted on the ERBs in each frequency region. Here, for example, the $\mathrm{CF}=913 \mathrm{~Hz} \mathrm{ERB}$ at the low test level was compared to the $\mathrm{CF}=1095 \mathrm{~Hz} \mathrm{ERB}$ at the same level for each of the 20 subjects. The same associations were measured in the $4000-\mathrm{Hz}$ region and data from both the low and high test levels were examined. Table VI contains the correlations between filter ERBs at neighbor-

TABLE V. Auditory-filter ERBs expressed as a proportion of the filter's CF.

\begin{tabular}{lcccc}
\hline \hline & $913 \mathrm{~Hz}$ & $1095 \mathrm{~Hz}$ & $3651 \mathrm{~Hz}$ & $4382 \mathrm{~Hz}$ \\
\hline Low level & & & & \\
$\quad$ Mean & 0.161 & 0.157 & 0.157 & 0.143 \\
s.d. & 0.022 & 0.023 & 0.021 & 0.020 \\
High level & & & & \\
$\quad$ Mean & 0.160 & 0.171 & 0.172 & 0.166 \\
s.d. & 0.019 & 0.038 & 0.039 & 0.035 \\
\hline \hline
\end{tabular}

TABLE VI. Correlations between ERBs of auditory filters centered at neighboring frequencies.

\begin{tabular}{ccc}
\hline \hline $\begin{array}{c}\text { Stimulus } \\
\text { level }\end{array}$ & $\begin{array}{c}1000-\mathrm{Hz} \\
\text { region }\end{array}$ & $\begin{array}{c}4000-\mathrm{Hz} \\
\text { region }\end{array}$ \\
\hline Low level & 0.41 & 0.13 \\
High level & $0.86^{\mathrm{a}}$ & $0.67^{\mathrm{a}}$ \\
\hline \hline${ }_{\mathrm{a} p<0.01 .}$ & &
\end{tabular}

ing frequencies. The correlations were far stronger at high test levels, similar to the trends reported above with regard to the filters' slopes.

\section{Auditory-filter symmetry}

Table VII contains means and standard deviations of the symmetry indices for each frequency and intensity condition. Generally, as the relative level of a filter increases, the filter becomes less symmetric; the low-frequency slope decreases and/or the high-frequency slope increases. The data reported here support previous findings relating the effect of masker level to increases in the SI (e.g., Formby, 1990; Sommers and Humes, 1993; Patterson et al., 1982). At three of the four test frequencies $(\mathrm{CF}=1095 \mathrm{~Hz}$ was the exception) the low-frequency slope was steeper than the high-frequency slope at the low masker level, while the opposite relation was noted at the high masker level at all four CFs.

A two-factor (frequency and level) ANOVA with repeated measures compared SIs across the different test conditions. The analysis indicated that only the main effect of level was significant $[\operatorname{GGF}(1,19)=88.17 ; p<0.001]$. Auditory filters were more asymmetric at the high signal level (70 dB SPL) regardless of stimulus frequency. The frequency at which the filter was centered was not significant $[\operatorname{GGF}(1,19)$ $=5.88 ; p>0.05]$, and there was no interaction between frequency and level $[\operatorname{GGF}(1,19)=2.49 ; p>0.05]$.

Two additional calculations of the slopes, ERBs, and correlations using a subset of the data were conducted in order to address reviewers' concerns that the five-point (Stone et al., 1992) method might not provide a stable measure of auditory-filter parameters, particularly the slope values. In these analyses, two individual runs from each subject were randomly selected and then analyzed as previously described. Therefore, three analyses were completed; the first utilized the mean data (reported above), while the last two used individual runs. In all cases, the same trends originally reported were confirmed (see the Appendix).

TABLE VII. Symmetry index (SI) derived by obtaining the quotient $P u / P l(N=20)$.

\begin{tabular}{lcccc}
\hline \hline & \multicolumn{4}{c}{ Auditory filter center frequency } \\
\cline { 2 - 5 } & $913 \mathrm{~Hz}$ & $1095 \mathrm{~Hz}$ & $3651 \mathrm{~Hz}$ & $4382 \mathrm{~Hz}$ \\
\hline Low level & & & & \\
$\quad$ Mean & 0.80 & 1.00 & 0.90 & 0.84 \\
s.d. & 0.19 & 0.22 & 0.26 & 0.27 \\
High level & & & & \\
$\quad$ Mean & 1.11 & 1.39 & 1.33 & 1.46 \\
$\quad$ s.d. & 0.31 & 0.34 & 0.31 & 0.36 \\
\hline \hline
\end{tabular}




\section{DISCUSSION}

An active feedback mechanism that utilizes a physiological analog to regenerative receivers has been theorized as a means by which the cochlea achieves its exquisite sensitivity and sharp tuning (Gold, 1948). Because cochlear nonlinearity saturates at moderate signal levels, the contribution to cochlear mechanics of such a process should be relatively greater when processing low-intensity rather than high-intensity sounds (e.g., Kim et al., 1980; Rhode, 1971; Zwicker, 1979). In addition to being displaced by a greater amplitude relative to the intensity of the input, the partition's shape is characterized, at the lower stimulus levels, as having a sharp peak at the point of maximum displacement (e.g., Dallos, 1992; Davis, 1983).

\section{A. Auditory-filter parameters}

\section{Relations between slopes of the auditory filters}

Auditory filters widened with increasing signal or masker level and the ERB was dictated by a broadening of the low-frequency skirt. The correlations in Table IV showed that low- and high-frequency slopes were dissimilar when compared at neighboring CFs at lower masker levels, particularly in the $4000-\mathrm{Hz}$ region (i.e., $P l$ at $\mathrm{CF}=3651 \mathrm{~Hz}$ with $P l$ at $\mathrm{CF}=4383 \mathrm{~Hz}$ ). The effect that masker level exerted upon the correlation could reflect the integrity of a vulnerable active feedback mechanism that contributed to cochlear frequency analysis relatively more at low signal levels. With low input level, the cochlear partition may require energy in addition to that provided by the stimulus to analyze most effectively the spectral components of the stimulus. Undamping of the basilar membrane movement is likely aided by the motile activity of outer hair cells (e.g., Brownell, 1986; Dallos, 1992). But as outer hair cells are among those cochlear structures most vulnerable to the effects of noise or drugs, it is possible that listeners could have discrete and highly localized regions in which the active processes could not be fully realized. Recall that all subjects in this research had normal absolute sensitivity not only at octave frequencies but at the filter CFs, as determined with the Bekesy technique.

Correlations were affected more by the signal level in the $4000-\mathrm{Hz}$ region than in the $1000-\mathrm{Hz}$ region. As demonstrated by Bekesy (1960), the passive basilar membrane is displaced by a smaller amount at the cochlea's base than at its apex, given similar stimulus input levels. Therefore, the possibility exists that an active, nonlinear effect would be strongest in that part of the cochlea requiring the greatest gain from an external source. However, when such a mechanism's operation is compromised locally, auditory filters could display quite different powers of frequency analysis across small differences in $\mathrm{CF}$.

In contrast, a relatively smaller contribution of the active feedback system (e.g., Whitehead et al., 1992; Zwicker, 1979) would be available to auditory filters operating at a higher relative level. As more passive basilar membrane mechanics are responsible for cochlear frequency analysis at high levels, the filters would appear more homogeneous across CFs for any given listener because each listener would rely upon essentially uniform passive inner ear structures.
That is, auditory filters measured in neighboring areas should be shaped more similarly when less dependent upon vulnerable processing mechanisms.

\section{Symmetry index}

The symmetry measure was different from the correlations between slopes of the auditory-filter skirts discussed above in that, for measures of auditory-filter symmetry, the comparison was between the skirt slopes of any one filter. The stronger stimuli at each $\mathrm{CF}$ yielded a low $P l$, resulting in an asymmetric auditory filter characterized by an SI $>1.0$. A similar finding was reported by Sommers and Humes (1993) when comparing the filters of hearing-impaired listeners to normal-hearing listeners who had hearing loss simulated by the introduction of external noise, and who were tested at a masker level comparable to the high level used in the present research $(\mathrm{No}=50 \mathrm{~dB})$. Although specific hypotheses for such a difference were not indicated, the data were consistent with a situation in which the basilar membrane displacement pattern was quite asymmetric, with displacement amplitude greater on the basal side than on the apical side of the peak displacement. The basal spread of excitation that accompanied the higher stimulus level in the current study also suggested the mechanics available to sharpen tuning contributed relatively less to frequency selectivity at high test levels. This situation was evinced by the filter asymmetry at high test levels.

\section{B. Relations between auditory-filter ERBs}

In light of previous investigations, two important trends were noted here with regard to measurements of ERBs at the different CFs and levels. First, increasing masker spectrum level produced significantly wider ERBs. The differences were found at all frequencies, save $913 \mathrm{~Hz}$, and were similar to those described by investigators such as Patterson (1976) and Weber (1977). Level effects in the current study were not as large as in Weber's, whose paradigm included several masker levels.

Glasberg and Moore (1990) suggested that changes in auditory-filter bandwidth as a function of masker level were due almost exclusively to changes in the low-frequency slope of the filter, or the high-frequency side of the displacement pattern. The shallower slopes found on the lowfrequency side of a filter's CF offset the observed slight sharpening of the filter's high-frequency skirt or apical side of the excitation pattern. Recall that the low-frequency slopes of the neighboring auditory filters were more strongly correlated than the high-frequency slopes (see Table III). The ERB change, which was influenced by increased masking at points basal to the peak displacement, coincided with the greater similarity between $\mathrm{Pl}$ 's found at high masker levels.

It is worth noting briefly that the auditory-filter skirts may be analogous to, or affected by, the pronounced absolute sensitivity variations found in threshold microstructure experiments (e.g., Long, 1984). Indeed, microstructure may have affected the data reported here by producing a narrow region (perhaps a high-dynamic range tip) that would appear sharply tuned because of an anomalous low unmasked 
threshold. The effects of microstructure diminish with increasing signal level, as do the differences between frequency selectivity when such function is assessed at neighboring CFs. An experiment that tests more directly the association between the two phenomena may confirm such an interdependence.

The aforementioned level effects displayed as changes in auditory-filter slope values were echoed in the ERB data. A particularly appealing aspect of the roex $(p, r)$ model relates to its treatment of $P u$ and $P l$, which are allowed to vary independently of one another. One consequence of this independence produced the striking similarity of $P l$ values at the high test level without a concurrent homogenizing of $P u$ values. It was unclear whether the ERBs, derived from both $P u$ and $P l$, would display the same level dependence when compared across neighboring CFs.

Consider first the difference in CFs in terms of ERB units (e.g, Moore and Glasberg, 1983). As pointed out by one reviewer, although the frequency ratio between neighboring CFs was the same in both regions (1.2), the difference expressed in ERB units was greater in the 4000-Hz region (1.6) than in the $1000-\mathrm{Hz}$ region (1.37). It should follow that the auditory filters centered in the $4000-\mathrm{Hz}$ region would be less similar than those centered at about 1000-Hz. In fact, Table VI shows just such a trend at both test levels. However, the level effect is stronger in the $4000-\mathrm{Hz}$ region, similar to the findings reported for the slope analysis. It is interesting to consider that, despite the separation in ERB units, the $P l$ values in the $4000-\mathrm{Hz}$ region are as well-correlated as measured here. As $P l$ corresponds to the basal side of the displacement pattern (e.g., Glasberg and Moore, 1990), the similarity of filter ERBs, particularly in the $4000-\mathrm{Hz}$ region, is driven by the similarity of basilar membrane displacement basal to the point of maximum displacement.

When both filter skirts are combined to produce an ERB measure, then, there is a distinct smoothing effect that appears to reduce variability displayed by the $P u$ and $P l$ values considered individually (Wright, 1996). As such, the ERB may be considered less informative than an analysis addressing differences between $P u$ and $P l$. It is clear from the data reported above that the effect of level on the association between filter bandwidths is produced, in large part, by the behavior of the filter's low-frequency skirt. As $P l$ reflects, albeit indirectly, the basal side of the basilar membrane's displacement, the similarity of filter parameters associated with high test level may be due, at least in part, to the level dependencies of the partition's displacement on the basal side of a signal's best place. Further, as level appears to exert a stronger influence on auditory-filter similarity in the $4000-\mathrm{Hz}$ region, there is likely a frequency dependence to the level effect.

\section{CONCLUSIONS}

(1) Auditory filters were found to be more asymmetric at high levels than at low levels. The level effect operated primarily by broadening the tuning of the filter's lowfrequency slope.

(2) In both the $1000-\mathrm{Hz}$ and $4000-\mathrm{Hz}$ frequency regions, $P l, P u$, and ERB were more similar across filter $\mathrm{CF}$ at high masker levels. This finding was attributed to the relatively smaller contribution of the active feedback mechanism apparent at high test intensities. The physiological vulnerability of such a mechanism is clear, and damage in the cochlea displaying normal absolute sensitivity may be restricted to a small area of the partition. When compared to an adjacent area, the difference in filtering ability may be quite obvious.

\section{ACKNOWLEDGMENTS}

B. R. Glasberg and B.C.J. Moore provided the software used in the fitting procedure. F. N. Martin, D. McFadden, D. C. Teas, and L. M. Thibodeau reviewed earlier drafts of this paper, and their efforts are still greatly appreciated. The text benefited substantially from the comments of Sid P. Bacon and an anonymous reviewer. These data were collected as part of a doctoral dissertation completed by the first author at the University of Texas at Austin. This work was supported (in part) by research Grant No. DC01419 from the National Institute on Deafness and Other Communication Disorders, a Jamail Grant from the University of Texas College of Communication, and by a generous contribution from the SERTOMA Club of Northwest Austin.

\section{APPENDIX}

Mean $(N=20)$ low- and high-frequency auditory-filter slopes with one standard deviation $(P l$ and $P u$, respectively). Data from the reanalyses were derived from subsets of the mean data.

$$
\begin{aligned}
& 913 \mathrm{~Hz} \quad 1095 \mathrm{~Hz} \quad 3651 \mathrm{~Hz} \quad 4382 \mathrm{~Hz} \\
& \begin{array}{lllllllll}
P l & P u & P l & P u & P l & P u & P l & P u
\end{array}
\end{aligned}
$$

Low level

$\begin{array}{lllllllll}\text { Mean } & 29.2 & 22.8 & 26.5 & 26.2 & 28.3 & 24.8 & 32.4 & 25.7\end{array}$ $\begin{array}{lllllllll}\text { Repeat No. } 1 & 30.2 & 24.9 & 26.5 & 26.0 & 31.1 & 25.1 & 34.0 & 26.3\end{array}$ $\begin{array}{lllllllll}\text { Repeat No. } 2 & 28.4 & 23.1 & 26.6 & 24.7 & 29.2 & 24.8 & 33.3 & 24.9\end{array}$ High level

$\begin{array}{lllllllll}\text { Mean } & 24.9 & 26.7 & 21.3 & 29.1 & 21.6 & 28.1 & 21.4 & 30.5\end{array}$ $\begin{array}{lllllllll}\text { Repeat No. } 1 & 25.3 & 25.8 & 21.1 & 31.0 & 21.3 & 28.0 & 21.2 & 29.4\end{array}$

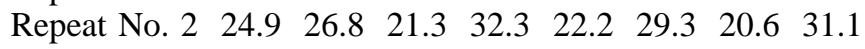

Mean auditory-filter ERB reanalyses (expressed as ERB/CF).

$\begin{array}{lcccc} & 913 \mathrm{~Hz} & 1095 \mathrm{~Hz} & 3651 \mathrm{~Hz} & 4382 \mathrm{~Hz} \\ \text { Low level } & & & & \\ \text { Mean } & 0.161 & 0.157 & 0.157 & 0.143 \\ \text { Repeat No. 1 } & 0.156 & 0.159 & 0.156 & 0.150 \\ \text { Repeat No. 2 } & 0.163 & 0.165 & 0.160 & 0.153 \\ \text { High level } & & & & \\ \text { Mean } & 0.160 & 0.171 & 0.172 & 0.166 \\ \text { Repeat No. 1 } & 0.165 & 0.167 & 0.176 & 0.171 \\ \text { Repeat No. 2 } & 0.161 & 0.170 & 0.172 & 0.172\end{array}$

Allen, J. B., and Fahey, P. F. (1992). "Using acoustic distortion products to measure the cochlear amplifier gain on the basilar membrane,' J. Acoust. Soc. Am. 92, 178-188.

ANSI (1989). ANSI S3.6-1989, “American National Standard specification for audiometers"' (American National Standards Institute, New York). Bekesy, G. von (1960). Experiments in Hearing (McGraw-Hill, New York). Brownell, W. E. (1986). "Outer hair cell motility and cochlear frequency selectivity,' in Auditory Frequency Selectivity, edited by B. C. J. Moore and R. D. Patterson (Plenum, New York), pp. 109-120. 
Dallos, P. (1992). "The Active Cochlea," J. Neurosci. 12, 4575-4585.

Davis, H. (1983). "'An active process in cochlear mechanics,' Hear. Res. 9, 79-90.

Fagelson, M. A., and Champlin, C. A. (1996). "Distortion-Product otoacoustic emissions and auditory-filter shape," Poster presentation at the 19th annual mid-winter meeting of the Association for Research in Otolaryngology, St. Petersburg, FL, 1996.

Formby, C. (1990). "Triangular approximations of auditory-filter shape," J. Speech Hear. Res. 33, 530-539.

Glantz, S. A., and Slinker, B. K. (1990). Primer of Applied Regression and Analysis of Variance (McGraw-Hill, New York).

Glasberg, B. R., and Moore, B. C. J. (1990). "Derivation of auditory-filter shapes from notched-noise data," Hear. Res. 47, 103-138.

Gold, T. (1948). "Hearing. The physical basis of the action of the cochlea," Proc. R. Soc. Edinburgh 135, 492-498.

Gold, T., and Pumphrey, R. J. (1948). "Hearing. The cochlea as a frequency analyzer," Proc. R. Soc. Edinburgh 135, 462-491.

Kim, D. O., Molnar, C. E., and Matthews, J. W. (1980). "Cochlear mechanics: Nonlinear behavior in two-tone responses as reflected in cochlearnerve responses and in ear-canal sound pressure,"' J. Acoust. Soc. Am. 67, $1704-1721$.

Levitt, H. (1971). “'Transformed up-down methods in psychoacoustics,' J. Acoust. Soc. Am. 49, 476-477.

Long, G. R. (1984). "The microstructure of quiet and masked thresholds," Hear. Res. 15, 73-87.

Margolis, R. H., and Shanks, J. E. (1985). “Tympanometry," in Handbook of Clinical Audiology, edited by J. Katz (Williams and Wilkins, Baltimore), pp. 438-475.

Mills, D. M., and Rubel, E. W. (1996). "Development of the cochlear amplifier,"' J. Acoust. Soc. Am. 100, 428-441.

Moore, B. C. J., and Glasberg, B. R. (1983). "Suggested formulae for calculating auditory-filter bandwidths and excitation patterns," J. Acoust. Soc. Am. 74, 750-753.

Pasanen, E. G., Wier, C. C., and McFadden, D. (1987). "Reciprocal relation between the growth of an emitted cubic distortion product and the suppression of a spontaneous otoacoustic emission," J. Acoust. Soc. Am. Suppl. 1 81, S8.

Patterson, R. D. (1976). "Auditory-filter shapes derived with noise stimuli,” J. Acoust. Soc. Am. 59, 640-654.
Patterson, R. D., Nimmo-Smith, I., Weber, D. L., and Milroy, R. (1982). "The deterioration of hearing with age: Frequency selectivity, the critical ratio, the audiogram and speech thresholds," J. Acoust. Soc. Am. 72, $1788-1803$.

Rhode, W. S. (1971). "Observations of the vibration of the basilar membrane in squirrel monkeys using the Mossbauer technique," J. Acoust. Soc. Am. 49, 1218-1231.

Rhode, W. S. (1977). "Some observations on two-tone interaction measured with the Mössbauer effect,"' in Psychophysics and Physiology of Hearing, edited E. F. Evans and J. P. Wilson (Academic London), pp. 27-37.

Robles, L., Ruggero, M. A., and Rich, N. C. (1986). "Basilar membrane mechanics at the base of the chinchilla cochlea. I. Input-output functions, tuning curves and response phases," J. Acoust. Soc. Am. 80, 1364-1374.

Rosen, S., and Baker, R. J. (1994). "Characterising auditory filter nonlinearity," Hear. Res. 73, 231-243.

Rosen, S., and Stock, D. (1992). "Auditory filter bandwidths as a function of level at low frequencies $(125 \mathrm{~Hz}-1000 \mathrm{~Hz})$," J. Acoust. Soc. Am. 92, 773-781.

Sommers, M. S., and Humes, L. E. (1993). "Auditory-filter shapes in normal-hearing, noise-masked normal, and elderly listeners," J. Acoust. Soc. Am. 93, 2903-2914.

Stone, M. A., Glasberg, B. R., and Moore, B. C. J. (1992). "Simplified measurement of auditory filter shapes using the notched-noise method," Br. J. Audiol. 26, 329-334.

Subramaniam, M., Henderson, D., and Spongr, V. (1994). “The relationship among distortion-product otoacoustic emissions, evoked potential thresholds, and outer hair cells following interrupted noise exposures," Ear Hear. 15, 299-309.

Weber, D. L. (1977). "Growth of masking and the auditory filter," J. Acoust. Soc. Am. 62, 424-429.

Whitehead, M. L., Lonsbury-Martin, B. L., and Martin, G. K. (1992). "Evidence for two discrete sources of 2F1-F2 distortion-product otoacoustic emission in rabbit. II: Differential physiological vulnerability," J. Acoust. Soc. Am. 92, 2662-2682.

Wright, B. A. (1996). Personal communication.

Zwicker, E. (1979). "A model describing nonlinearities in hearing by active processes with saturation at $40 \mathrm{~dB}$," Biol. Cybern. 35, 243-250. 